\title{
Polimorfismos del gen del receptor de vitamina $D$ y riesgo de fractura de cadera en la mujer adulta mayor de la Región del Bío Bío
}

\author{
Iván Q uevedo L, Milka Martínez $\mathrm{B}^{\mathrm{a}}$, Marcelo Castillo N ${ }^{\mathbf{b}}$, \\ Nancy Rivera $F^{b}$.
}

\author{
Vitamin $D$ receptor gene \\ polymorphisms and risk of hip \\ fracture in Chilean elderly women
}

Background: Osteoporotic hip fractures are devastating events in older women. There is a genetic modulation of bone phenotypic parameters including bone density (BMD) and bone fragility fractures. Vitamin D receptor (VDR) gene polymorphisms explain a small part of the genetic influence on BMD, whereas their effect on fractures remains uncertain. Aim: To examine the contributions of VDR genotypes to the susceptibility to hip fracture in elderly Chilean women. Patients and methods: We recruited 126 women (67 with fractures and 59 without) from Bio-Bio Region, Chile, aged 65 to 94 years. Genotyping for Bsm-1, Apa-1, Taq-1 and Fok-1 VDR polymorphisms was performed using polymerase chain reaction methods. All hip fractures were confirmed by X-ray. Results: The allele frequencies were 0.49 for B, 0.57 for A, 0.60 for T and 0.65 for F in the Bsm-1, Apa-1, Taq-1 and Fok-1 polymorphisms respectively. The prevalence of these VDR gene polymorphisms in women with fractures were $16 \% \mathrm{BB}, 69 \% \mathrm{Bb}, 15 \%$ bb for Bsm- $1 ; 30 \% \mathrm{AA}$, $46 \%$ Aa, $14 \%$ aa for Apa-1; $17 \%$ TT, 34 Tt, $8 \%$ tt for Taq-1 and 43\% FF, 41\% Ff, 16\% ff for Fok-1. All VDR genotype frequencies did not differ from Hardy-Weinberg expectations. Allele or genotype frequencies did not differ between women with or without fractures. These results did not change when analysis was adjusted by age weight, height or gynecologic history. Conclusions: The genotype frequencies of the VDR polymorphisms are in accordance with the frequencies of other Hispanic and Caucasian populations. Our results suggest that VDR polymorphisms are not associated with the risk of hip fracture in older women of this Region of Southern Chile (Rev Méd Chile 2008; 136: 475-81). (Key words: Hip fractures; Receptors, calcitriol; Osteoporosis, postmenopausal)

\footnotetext{
Recibido el 13 de julio, 2007. Aceptado el 11 de diciembre, 2007.

Este estudio fue financiado a través del Proyecto DIUC \# 205.085.030.1-0 de la Dirección de Investigación de la Universidad de Concepción y Departamento de Estudios Clínicos de Laboratorios SAVAL S.A.

Laboratorio de Medicina Molecular, Dirección de Investigación, Facultad de Medicina Universidad de Concepción.

aioquímico

${ }^{\mathrm{b}}$ Tecnólogo Médico
}

Correspondencia a: Dr. Iván Quevedo L. Laboratorio de Medicina Molecular, Dirección de Investigación de la Facultad de Medicina, Universidad de Concepción. Janequeo 151, Concepción. Fax: (56-41) 223 2003. E mail: equevedo@udec.cl 
$\mathrm{L}$ a osteoporosis es una enfermedad esquelética sistémica, caracterizada por una baja masa ósea y deterioro en la microarquitectura del tejido óseo, que origina fragilidad ósea aumentada con el consecuente aumento en el riesgo de fractura ${ }^{1}$. Se considera actualmente que la osteoporosis es la patología crónica no transmisible de más rápido crecimiento en el mundo y la patología crónica más prevalente en las mujeres mayores de 65 años $^{2}$. Para las mujeres caucásicas de origen anglosajón mayores de 60 años el riesgo de fractura osteoporótica en el período restante de sus vidas será de 60\% y de alrededor de 30\% para los hombres de la misma edad ${ }^{3}$. Las fracturas osteoporóticas en Estados Unidos de Norteamérica, desde hace 10 años, tienen un costo superior a los 14 billones de dólares anuales ${ }^{4}$. La más temida de las fracturas osteoporóticas es la fractura de cadera, dado que tiene una elevada mortalidad durante el primer año de ocurrida (12\% a 20\%), la mitad de los sobrevivientes requerirá asistencia para caminar y 25\% necesitará atención institucionalizada permanente ${ }^{5}$. En Chile, dos estudios realizados durante la década pasada, mostraron un incremento en la tasa de fractura de caderas, especialmente en los mayores de 70 años $^{6-7}$, siendo la edad promedio de las fracturas de cadera de 71,5 años en las mujeres y 74,8 años en los hombres ${ }^{8}$.

La osteoporosis es una enfermedad multifactorial en la que participan elementos ambientales, nutricionales y genéticos. El conocimiento progresivo de estos factores de riesgo, más la información que entrega la densitometría ósea permitirán predecir con mayor certeza el riesgo de fractura? Diversos estudios en miembros de una misma familia y en gemelos muestran que los factores genéticos juegan un papel importante en la regulación de la masa ósea y de otras características del hueso, que se traducen en una susceptibilidad aumentada para la fractura osteoporótica. La importancia de los factores genéticos en la determinación de la masa ósea ha sido estimada en alrededor de 50\%. Consecuencia de la anterior observación, múltiples estudios en los genes implicados en la homeostasis y estructura del hueso han sido realizados en los genes del receptor de estrógenos, colágeno tipo 1, citoquinas y factores de crecimiento, pero sobre todo del gen de receptor de vitamina D (VDR). La asocia- ción de un polimorfismo del gen de VDR con el recambio óseo fue descrita por primera vez en 1992, por Morrison et al. En ese estudio realizado en una población de origen anglosajón, el genotipo BB del polimorfismo Bsm 1 fue significativamente mayor en personas con elevados niveles plasmáticos de marcadores del recambio óseo ${ }^{10}$. Luego del estudio de Morrison, múltiples trabajos en diferentes poblaciones alrededor del mundo han mostrado resultados contradictorios referentes a la asociación del polimorfismo Bsm 1, y de otros polimorfismos del VDR posteriormente descritos, con osteoporosis. Incluso, el último metaanálisis publicado mostró sólo un efecto marginal de los polimorfismos de VDR en la variación de la densidad mineral ósea (DMO). Si bien la DMO es uno de los principales determinantes del riesgo de fractura aproximadamente la mitad de los pacientes con fractura osteoporótica tienen una DMO bajo los criterios para el diagnóstico de osteoporosis según la OMS. Reforzando esta observación, un reciente estudio prospectivo en mujeres posmenopáusicas francesas mostró que los polimorfismos Bsm 1, Apa 1 y Taq 1 se asociaron con un mayor riesgo para fractura osteoporótica independientemente de la DMO, niveles plasmáticos hormonales y de los marcadores bioquímicos de recambio óseo ${ }^{11}$.

Siguiendo esta última línea de trabajo el propósito de nuestro estudio fue determinar si los polimorfismos Bsm 1, Apa 1, Taq 1 y del polimorfismo Fok 1 del VDR se asocian a un mayor riesgo de fractura osteoporótica de cadera en la población adulta mayor femenina de la Región del Bío Bío.

\section{PACIENTES Y MÉTODO}

Pacientes. Se estudiaron 138 mujeres adultas mayores provenientes de la Región del Bío Bío, que consultaron en el Hospital Traumatológico de Concepción durante el año 2004. Setenta y cuatro pacientes ingresaron por fractura de cadera y 64 pacientes, consideradas como grupo control, consultaron por lumbago crónico en el mismo recinto asistencial. Se confirmó en todas las pacientes el diagnóstico de fractura de cadera y de lumbago crónico a través de un estudio radiológico. Dado que el objetivo de nuestro trabajo fue determinar si los polimorfismos Bsm 1, Apa 1, Taq 1 y Fok 1 
del VDR se asociaban a un mayor riesgo de fractura de cadera independientemente de la DMO no se realizó en la población estudiada un estudio densitométrico óseo. A todas las mujeres se les realizó una entrevista con el fin de obtener los datos respecto a edad de la menarquia, número de hijos, menopausia, estrogenoterapia o medicamentos que pudiesen afectar el metabolismo óseo tales como glucocorticoides o vitamina D. Además con el fin de descartar patologías que pudiesen afectar la masa ósea se les realizó una evaluación clínica y bioquímica cuyos resultados se muestran en la Tabla 1.

A todas las pacientes con fractura de cadera y del grupo control se les solicitó el consentimiento informado para participar en el estudio de acuerdo a las normas de la Declaración de Helsinki y el protocolo fue aprobado por el Comité de Ética de la Dirección de Investigación de la Facultad de Medicina de la Universidad de Concepción.

Fueron excluidas 12 mujeres del estudio ( 7 con fractura de cadera y 5 del grupo control), que tenían insuficiencia renal crónica (4), hipercalcemia (1), estrogenoterapia por más de 6 meses (2), insuficiencia hepática (2) y corticoterapia crónica (3).

Genotipificación. Se extrajo el ADN genómico a partir de leucocitos de sangre periférica de las pacientes y controles usando un procedimiento de extracción estándar no enzimático ${ }^{12}$. El análisis genético del polimorfismo Fok 1 del exón 2 se realizó por amplificación con partidores que fue- ron previamente descritos ${ }^{13}$. Las reacciones de PCR se realizaron en un termociclador Mastercicler $^{\circledR}$ Eppendorf utilizando aproximadamente 0,5 $\mu \mathrm{g}$ de ADN. La denaturación inicial fue a $94^{\circ} \mathrm{C}$ por $5 \mathrm{~min}$, seguido de 35 ciclos a $94^{\circ} \mathrm{C}$ por $20 \mathrm{~s}$, un apareamiento a $60^{\circ} \mathrm{C}$ durante $30 \mathrm{~s}$, polimerización a $72^{\circ} \mathrm{C}$ durante $30 \mathrm{~s}$ y polimerización final durante 7 min a $72^{\circ} \mathrm{C}$. Los productos de la PCR fueron digeridos por la enzima de restricción Fok 1 (New England Biolabs) a $37^{\circ} \mathrm{C}$ por $120 \mathrm{~min}$.

Los análisis genéticos de los polimorfismos intrónicos Apa 1 y Taq 1 se realizaron con el partidor sentido (5' CAG AGC ATG GAC AGG GAG CAA G 3') y el partidor antisentido (5' GCA ACT CCT CAT GGC TGA GGT CTC A 3'). Después de la denaturación inicial a $94^{\circ} \mathrm{C}$ se continuó por 5 min de 30 ciclos a $94^{\circ} \mathrm{C}$ por $40 \mathrm{~s}$, apareamiento a $60^{\circ} \mathrm{C}$ por $40 \mathrm{~s}$, polimerización a $72^{\circ} \mathrm{C}$ por $40 \mathrm{~s}$ con polimerización final a $72^{\circ} \mathrm{C}$ por $7 \mathrm{~min}$. El fragmento producido de $\mathrm{ADN}$ de $745 \mathrm{pb}$ fue digerido con ambas enzimas de restricción Taq 1 (New England Biolabs) a $65^{\circ} \mathrm{C}$ por 90 min o Apa 1 (New England Biolabs) a $37^{\circ} \mathrm{C}$ por $120 \mathrm{~min}$.

La región polimórfica Bsm 1 fue amplificada utilizando el partidor sentido (5' CAA CCA AGA CTA CAA GTA CCG CGT CAG TGA 3') y el partidor antisentido (5' AAC CAG CGG AAG AGG TCA AGG G 3'). El fragmento originado fue de $825 \mathrm{pb}$, el que fue sometido a idénticas condiciones que los polimorfismos Apa $1 \mathrm{y}$ Taq 1. Los productos de la PCR fueron digeridos con la enzima Bsm 1 (New England Biolabs) a $65^{\circ} \mathrm{C}$ por $120 \mathrm{~min}$.

Tabla 1. C aracterísticas clínicas y bioquímicas de las pacientes estudiadas

\begin{tabular}{|c|c|c|c|}
\hline & $\begin{array}{l}\text { Fractura de cadera } \\
\qquad n=67\end{array}$ & $\begin{array}{c}\text { Controles } \\
\mathrm{n}=59\end{array}$ & $\mathrm{p}$ \\
\hline Edad (años) & $77 \pm 4$ & $78 \pm 9$ & 0,08 \\
\hline Talla (cm) & $152 \pm 6$ & $153 \pm 7$ & 0,68 \\
\hline Peso (kg) & $62 \pm 11$ & $60 \pm 12$ & 0,14 \\
\hline Hijos (n) & $5,1 \pm 3,3$ & $5,3 \pm 3,3$ & 0,82 \\
\hline Menarquia (años) & $13,7 \pm 1,4$ & $13,7 \pm 1,5$ & 0,88 \\
\hline Creatininemia $(\mathrm{mg} / \mathrm{dl})$ & $1,0 \pm 0,3$ & $0,9 \pm 0,2$ & 0,06 \\
\hline Calcemia (mg/dl) & $8,8 \pm 0,6$ & $8,7 \pm 0,5$ & 0,49 \\
\hline Glicemia (mg/dl) & $105 \pm 31$ & $102 \pm 33$ & 0,71 \\
\hline Fosfatasas alcalinas totales (UI) & $206 \pm 54$ & $138 \pm 33$ & $<0,001$ \\
\hline
\end{tabular}


Identificación de los alelos. Los productos de la PCR se sometieron a una electroforesis en un gel de agarosa al 1\%. Para los polimorfismos Bsm 1, Apa 1 y Taq 1. Se utilizó un gel de agarosa para la identificación del polimorfismo Fok 1. Luego, los geles se tiñeron en una solución de bromuro de etidio y el resultado se visualizó en un transiluminador de luz ultravioleta. Según la presencia o ausencia del sitio de restricción fueron identificados como BB (825 pb), Bb (650 pb) y bb (175 pb) para Bsm 1. En los casos de Apa 1 y Taq 1 fueron identificados AA (745 pb), Aa (528 pb), aa (217 pb) y TT (745 pb), Tt (450 pb), tt (295 pb). Por último, la identificación de los alelos en el polimorfismo Fok 1 fue FF (265 pb), Ff (196 pb) y ff (69 pb). Se comparó el tamaño de las bandas correspondiente en los amplicones que contienen los polimorfismos según su migración electroforética, usando estándares de peso molecular conocidos.

Estadística. Para el análisis se usó el programa SigmaStat 13.0 software (SPSS Inc., Illinois USA), presentándose estadísticas descriptivas (promedios \pm desviación estándar). La comparación entre grupos se hizo con el test de t de Student. Para eliminar potenciales diferencias confundentes relacionadas con las variables estudiadas se realizó un análisis de varianzas y de co-varianzas. En las pacientes con fractura de cadera y en las del grupo control se realizó un análisis de HardyWeinberg. Se consideraron estadísticamente significativos valores de significancia $\mathrm{p} \leq 0,05$.

\section{Resultados}

De las 138 mujeres estudiadas fueron genotipificadas 126, ya que cumplieron todos los criterios de inclusión para el estudio final. Al comparar ambos grupos de mujeres encontramos que la edad, peso y las variables ginecoobstétricas fueron similares. Dentro del estudio bioquímico realizado se encontró una elevación significativamente mayor $(p<0,001)$ de las fosfatasas alcalinas totales en el grupo con fractura de cadera, probablemente debido a un incremento de la actividad osteoblástica como respuesta a la fractura.

Se detectaron e identificaron todos los alelos objetos de estudio en las mujeres con fractura de cadera y del grupo control.
Estudio de asociación de Fok 1, Bsm 1, Apa 1 y Taq 1 del VDR.

Las frecuencias genotípicas en la cohorte de pacientes con fractura de cadera fueron $43 \% \mathrm{FF}$, $41 \% \mathrm{Ff}$ y $16 \% \mathrm{ff}$. En el grupo control las frecuencias fueron $46 \% \mathrm{FF}, 42 \%$ Ff y $12 \%$ ff. Las frecuencias alélicas en las mujeres estudiadas fueron 0,65 para $\mathrm{F}$ y 0,35 para $\mathrm{f}$. Ni el alelo $\mathrm{F}$ ni el $\mathrm{f}$ se asociaron con una mayor ocurrencia de fractura de cadera. En las 126 mujeres, las frecuencias alélicas en el estudio del polimorfismo Bsm 1, fueron 0,49 para B y 0,51 para b. Las frecuencias genotípicas en el grupo con fractura de cadera fueron $16 \% \mathrm{BB}, 69 \% \mathrm{Bb}$ y $15 \% \mathrm{bb}$. Respecto a la asociación de los polimorfismos Apa 1 y Taq 1 del VDR, en el grupo control las frecuencias genotípicas fueron $30 \% \mathrm{AA}, 46 \% \mathrm{Aa}, 14 \%$ aa y $17 \%$ TT, $34 \% \mathrm{Tt}, 8 \% \mathrm{tt}$, respectivamente. Las frecuencias genotípicas fueron 0,57 para el alelo A, 0,43 para b, 0,60 para $\mathrm{T}$ y 0,40 para $t$ no encontrándose asociación entre fractura de cadera y los polimorfismos Bsm 1, Apa 1 y Taq 1. Los resultados de las frecuencias genotípicas y distribuciones alélicas se muestran en la Tabla 2. La ley de equilibrio genético de Hardy-Weingber se cumplió para los grupos en estudio.

\section{DisCusión}

Los resultados de este trabajo muestran que todos los alelos de los polimorfismos Fok 1, Bsm 1, Apa 1 y Taq 1, ubicados en el codón de inicio, intrón 8 , exones 2 y 9 , del gen del receptor de vitamina D están presentes en la población adulta mayor de la Región del Bio Bío. La distribución de los genotipos de los diferentes polimorfismos del VDR en nuestro estudio fueron similares para el caso de Fok 1 a la encontrada en las mujeres caucásicas premenopaúsicas norteamericanas y francesas así como en la población general femenina de suiza y japonesa, pero muy diferente a $65 \% \mathrm{FF}, 31 \% \mathrm{Ff}$ y $4 \% \mathrm{ff}$ de las mujeres adultas afroamericanas de Estados Unidos de Norteamérica. Respecto a las distribuciones de los polimorfismos Bsm 1, Taq 1 y Apa 1 observados en nuestra población, éstos se asemejan a las de las poblaciones caucásicas de las poblaciones europeas y a la de las poblaciones de origen hispano de Estados Unidos de Norteamérica y Brasil, siendo significa- 
Tabla 2. Frecuencia genotípica y alélica de los polimorfismos Fok 1, Bsm 1, Apa 1, Taq 1 en pacientes con fractura de cadera y grupo control

\begin{tabular}{|c|c|c|c|c|c|c|}
\hline Polimorfismo & Genotipo & Alelos & $\begin{array}{c}\text { Fractura de cadera } \\
n=67(\%)\end{array}$ & $\begin{array}{c}\text { Control } \\
\mathrm{n}=59(\%)\end{array}$ & $\chi^{2}$ & $\mathrm{p}$ \\
\hline Fok 1 & $\begin{array}{l}\text { F } 65 \% \\
\text { f } 35 \%\end{array}$ & $\begin{array}{l}\mathrm{FF} \\
\mathrm{Ff} \\
\mathrm{ff}\end{array}$ & $\begin{array}{l}29(43) \\
27(40) \\
11(17)\end{array}$ & $\begin{array}{r}27(46) \\
25(42) \\
7(12)\end{array}$ & 0,53 & 0,77 \\
\hline Bsm 1 & $\begin{array}{l}\text { B } 49 \% \\
\text { b } 51 \%\end{array}$ & $\begin{array}{l}\mathrm{BB} \\
\mathrm{Bb} \\
\mathrm{bb}\end{array}$ & $\begin{array}{l}11(16) \\
46(69) \\
10(15)\end{array}$ & $\begin{array}{r}9(15) \\
37(63) \\
13(22)\end{array}$ & 1,06 & 0,59 \\
\hline Apa 1 & $\begin{array}{l}\text { A } 57 \% \\
\text { a } 43 \%\end{array}$ & $\begin{array}{l}\text { AA } \\
\text { Aa } \\
\text { aa }\end{array}$ & $\begin{array}{l}25(37) \\
31(46) \\
11(17)\end{array}$ & $\begin{array}{l}18(30) \\
27(46) \\
14(24)\end{array}$ & 1,27 & 0,53 \\
\hline Taq 1 & $\begin{array}{l}\text { T } 60 \% \\
\text { t } 40 \%\end{array}$ & $\begin{array}{l}\mathrm{tt} \\
\mathrm{Tt} \\
\mathrm{tt}\end{array}$ & $\begin{array}{l}26(39) \\
31(46) \\
10(15)\end{array}$ & $\begin{array}{r}17(29) \\
34(58) \\
8(19)\end{array}$ & 1,74 & 0,42 \\
\hline
\end{tabular}

tivamente diferentes a la de la población femenina de Japón. No encontramos una relación estadísticamente significativa que pudiese identificarse, en los polimorfismos estudiados, entre las poblaciones adultas mayores con fracturas de cadera y la población control.

Existen datos controversiales en la literatura médica respecto a los resultados que asocien un genotipo del VDR a fractura osteoporótica. Dos estudios prospectivos caso-control en mujeres posmenopaúsicas resultaron conflictivos, ya que en el estudio Nurses' Health el genotipo BB del VDR fue asociado con el doble del riesgo para fractura de cadera, pero no con fractura distal del radio. Por otro lado el estudio Osteoporotic Fractures en adultas mayores no encontró asociación entre ninguno de los genotipos del VDR y riesgo de fractura de cadera, vértebra u otro tipo de fractura no vertebral. Por otro lado, en el estudio Rotterdam, se reportó una significativa asociación entre los haplotipos del gen de VDR con riesgo de fractura de cadera y radio, ocurridas durante los 3,8 primeros años en promedio de seguimiento. Sin embargo, este mismo estudio con un seguimiento más prolongado a los 7 años en promedio, no encontró esta asociación en la misma pobla- ción. Otros estudios realizados en Bélgica y el Reino Unido no lograron determinar ninguna diferencia entre el genotipo de VDR con riesgo de fractura osteoporótica de cadera o masa ósea disminuida.

Recientemente dos estudios prospectivos, independientes entre sí realizados en Australia y Francia concluyeron que los polimorfismos Taq 1 y Bsm 1 se asocian significativamente a un incremento del riesgo de fractura de cadera y esta asociación fue independiente de la masa ósea, marcadores de recambio óseo, niveles hormonales o la edad. Ambos estudios plantean que los mecanismos a través de los cuales los genotipos del VDR influyen en la resistencia del hueso no están aclarados.

Existen varias posibles explicaciones para nuestros hallazgos: (a) El número de individuos en nuestro estudio puede ser pequeño y no tuvo el poder estadístico suficiente para demostrar una asociación. Sin embargo, consideramos que si hubiese una fuerte asociación entre los polimorfismos del VDR y fractura de cadera en nuestra población éstas habrían quedado en evidencia a lo menos reflejándose en una tendencia. (b) El hecho que nuestra población no sea étnicamente 
homogénea podría explicar nuestros resultados negativos. Dado lo anterior con el fin de evitar dentro de lo posible esta variable, la población estudiada tenía ambos apellidos de origen hispano, y provenía de los tres quintiles más pobres, excluyéndose pacientes con apellido de origen mapuche o extranjero. (c) Finalmente, se podría considerar que los alelos del VDR no influyen directamente en el riesgo de fractura de cadera, pero están en desequilibrio de ligamiento con alelos de otros genes vecinos relacionados con el metabolismo óseo.

\section{REFERENCIAS}

1. Kanis JA, Melton LJ, Christiansen C, Johnston CC, Khaltaev. The diagnosis of osteoporosis. J Bone Miner Res 1994; 9: 1137-41.

2. Jones G, Neuyen T, Sambrook PN, Kelly PJ, Gilbert C, Fisman JA. Symptomatic Fracture Incidence in elderly men and woman: The dubbo osteoporosis epidemiology stay (DOES). Osteoporosis Int 1994; 4: 277-82.

3. Cumming RG, Nevitt MC, Cummines SR. Epidemiology of the hip fractures. Epidemiol Rev 1997; 19: 244-57.

4. Contreras L, Kirschbaum A, Pumarino H. Epidemiología de las fracturas en Chile. Rev Méd Chile 1991; 119: 92-8.

5. Pumarino H, Contreras L, Kirschbaum A. Tendencia de las tasas de fractura de cadera en 12 años en Chile. ¿Aumento independiente del envejecimiento poblacional? Rev Méd Chile 1997; 125: 893-8.

6. Valdivia C, Giaconi J, Arteaga E, Pumarino H, Gajardo H, Villarroel L. Fractura de la cadera: Estudio de casos y controles en la Región Metropolitana. Rev Méd Chile 1996; 124: 189-97.

7. Kanis JA, Johnello, Oden A, De Laet C, Johnsson B, Dawson A. Ten-year risk of osteoporotic fracture on screening strategies. Bone 2002; 30: 251-8.

8. Johnston CC, Slemenda CW. Pathogenesis of postmenopausal osteoporotic fractures. In: Steven son JC, Lindsay R, Eds. Osteoporosis. Chapman \& Hall; London, 1998; 53-64.

9. Morrison NA, Qi JC, Tohita A, Kelly PJ, Crofts L, Nguyen TV et al. Prediction of bone density from vitamin D receptor alleles. Nature 1994; 367: 284-7.

10. Vitterlinden A, Ralston S, Brandi ML, Carey A, Grinberg D, LancDahl B et al. The association
En resumen en este trabajo hemos estudiado los polimorfismo Bsm 1, Apa 1, Taq 1 y Fok 1 del gen de VDR en una población adulta mayor femenina de la Región del Bio Bío con fractura osteoporótica de cadera. Los resultados muestran que en nuestra población están presente todos los polimorfismos del VDR estudiados y que su distribución genotípica se asemeja a la de las poblaciones europeas, brasileña y norteamericana de origen hispano. Sin embargo, los genotipos del VDR no estarían asociados con un mayor riesgo de fractura de cadera en nuestra población adulta mayor.

between common vitaminin $\mathrm{D}$ receptor gene variations an osteoporosis: A participant-level meta-analysis. Ann Intern Med 2006; 145: 255-64.

11. Garnero P, Muñoz F, Borel O, Sornay-Rendu E, Delmas P. Vitamin D receptor gene polymorphis$\mathrm{ms}$ are associated with the risk of fractures in postmenopausal women, independently of bone mineral density. J Clin Endocrinol Metab 2005; 90: 4829-35.

12. Gross C, Eccleshall TR, Malley PJ, Villa ML, Marcus R, Feldman D. The presence of a polymorphism at the translation initiation site of the vitamin $\mathrm{D}$ receptor gene is associated with low bone mineral density in postmenopausal Mexican-American women. J Bone Miner Res 1996; 11: 1043-8.

13. Ferrari S, Rizolli R, Manen D, Slosman D, Bonjour JP. Vitamin D receptor gene stars codon polymorphisms (FokI) and bone mineral density: Interaction with age, dietary calcium, and 3' end region polymorphisms. J Bone Miner Res 1998; 13: 925-30.

14. Arai $\mathrm{H}$, Miyamoto $\mathrm{K}$, Taketami $\mathrm{Y}$, Yamamoto $\mathrm{H}$, IEMORI Y, MORITA K ET AL. A vitamin D receptor gene polymorphism in the translation initiation codon: Effect on protein activity and relation to bone mineral density in Japanese Women. J Bone Miner Res 1997; 12: 915-21.

15. Zmuda J, Cauley J, Danielson M, Theobald T, Ferrell R. Vitamin D receptor translation imitiation codon polymorphism and markers of osteoporotic risk in older African. American women. Osteoporosis Int 1999; 9: 214-19.

16. Feskanich D, Hunter DJ, Willes WC, Hankinson JE, Hollis Bw, Hough HL et al. Vitamin D receptor genotype and the risk of bone fractures in women. Epidemiology 1998; 9: 535-9. 
17. Lazaretti-Castro M, Duarte de Oliveira Ma, Russo EM, VIEIRA JG. Vitamin D receptor alleles and bone density in a normal premenopausal Brazilian female population. Brazilian Journal of Medical and Biological Research 1997; 30: 929-32.

18. Fescanich D, Hunter DJ, Willet WC, Hankinson SE, Hollis BW, Hough HL et al. Vitamin D receptor gene polymorphisms and the risk of bone fractures en women. Epidemiology 1998; 9: 535-9.

19. Ensrud Ke, Stone K, Cauley JA, White C, Zmuda JM, NGUYEN TV ET AL. Vitamin D receptor gene polymorphisms and the risk of fractures in older women. For the study of fractures research group. J Bone Mineral Res 1999; 14: 1637-45.

20. Uitterlinden AG, Weel AEAM, Buerger H, Fang Y, Van Duijn CM, Hofman A et al. Interaction between the vitamin $\mathrm{D}$ receptor gene and collagen type I \& 1 gene in susceptibility for fracture. J Bone Miner Res 2001; 16: 379-85.

21. Colin EM, Uitterlinden AJ, Meurs JBJ, Bergink AP, VAN DE KLIFT M ET AL. Interaction between vitamin $\mathrm{D}$ receptor genotype and estrogen receptor $\mathrm{A}$ Genotype influences in vertebral fracture risk. J Clin Endocrinol Metab 2003; 88: 377-84.

22. Aerssens J, Dequeker J, Peeters J, Breemans S, Broos P, Boonen S. Polymorphisms of the VDR, ER and Colia 1 genes and osteoporotic hip fracture in elderly postmenopausal women. Osteoporosis Int 2000; 11: 583-91.

23. Nguyen TV, Esteban LM, White CP; Grant SF, Center JR, Gardiner EM et al. Contribution of the collagen I 1 and vitamin $\mathrm{D}$ receptor genes to the risk of hip fracture in elderly women. J Clin Endocrinol Metab 2005; 90: 6575-9. 